\title{
Optimized renormalization group flows
}

\author{
Daniel F. Litim* \\ Theory Division, CERN, CH-1211 Geneva 23, Switzerland \\ (Received 10 May 2001; published 12 October 2001)
}

\begin{abstract}
We study the optimization of exact renormalization group (ERG) flows. We explain why the convergence of approximate solutions towards the physical theory is optimized by appropriate choices of the regularization. We consider specific optimized regulators for bosonic and fermionic fields and compare the optimized ERG flows with generic ones. This is done up to second order in the derivative expansion at both vanishing and nonvanishing temperature. We find that optimized flows at finite temperature factorize. This corresponds to the disentangling of thermal and quantum fluctuations. A similar factorization is found at second order in the derivative expansion. The corresponding optimized flow for a "proper-time renormalization group" is also provided to leading order in the derivative expansion.
\end{abstract}

DOI: 10.1103/PhysRevD.64.105007

PACS number(s): 05.10.Cc, 11.10.Gh, 11.10.Kk, 11.10.Wx

\section{INTRODUCTION}

Wilsonian renormalization group techniques $[1,2]$ such as the exact renormalization group (ERG) [3-6] are important tools for addressing nonperturbative problems within quantum field theory (for recent reviews, see Refs. [7,8]). They are similar in spirit to the block-spin action invented in condensed matter physics, and their particular strength is their flexibility, allowing for systematic approximations without being tied to the small coupling region. The ERG is based on an infrared (IR) regularization with the momentum scale parameter $k$ of the full propagator, which turns the corresponding effective action into a scale dependent functional $\Gamma_{k}$. The ERG flow describes the change of the effective action under an infinitesimal variation of the IR scale $k$. It thereby interpolates between the initial UV action $\Gamma_{k=\Lambda}$ and the full quantum effective action $\Gamma \equiv \Gamma_{k=0}$. Although the flow depends explicitly on the specific infrared regulator chosen, the end point of the integrated full flow does not.

An explicit computation of the IR effective theory based on the ERG flow requires the specification of the field content, the initial condition $\Gamma_{\Lambda}$ and the choice of a particular IR regulator. The UV initial condition is typically given by the classical action. Hence, the main physical information is contained in the ERG flow itself. Most problems of physical interest are too complex to be solved exactly and an application of this formalism - as of any other method-is bound to certain approximations. Furthermore, the flow equation is equivalent to infinitely many coupled partial differential equations, which would seem very difficult to solve exactly. Therefore, one has to resort to some approximations or truncations which allow, at least in principle, for a systematic computation of the full quantum effective action. In order to provide reliable physical predictions, such as a high precision computation of universal critical exponents, it is mandatory to provide a good control for approximated ERG flows.

A number of systematic expansion schemes for flow equations are known, including standard perturbation theory.

*Electronic address: Daniel.Litim@cern.ch
Non-perturbative expansions of the effective action, not bound to the weakly coupled regime, are the derivative expansion, expansions in powers of the fields, or combinations thereof. For example, the leading order of the derivative expansion retains only an effective potential and a standard kinetic term, and contains non-perturbative information as it corresponds to the resummation of infinitely many perturbative loop diagrams. The study of approximate quantum effective actions along these lines is a sensible procedure since the underlying expansions admit a systematic improvement to higher order.

Solutions to truncated flow equations display a spurious dependence on the IR regulator [9-16]. This is similar to the scheme dependence of physical observables observed within perturbative QCD [17], or the truncation dependence of solutions to Schwinger-Dyson equations. Its origin is the following. The IR regulator couples, through the flow equation, to all vertex functions of the theory. The flow trajectory of the functional $\Gamma_{k}$ in the space of all effective action functionals depends on the regulator. Hence, the regulator-while regulating the flow-also modifies the effective interactions at intermediate scales $k \neq 0$. In other words, the effective action at intermediate scales still has some memory of the details of how the integrating-out of degrees of freedom has been performed. This regulator dependence is of no relevance for the full flow. Eventually, the convergence towards the full quantum effective action for any regulator ensures that all regulator-induced interactions cancel out in the physical limit. Approximations imply that certain vertex functions are neglected. Then, not all regulator-induced interactions cancel out for $k \rightarrow 0$ : the missing back coupling of the neglected vertex functions is responsible for regulatordependent terms in the physical limit. In consequence, approximations to the full quantum effective action depend spuriously on the scheme.

Recently, a new line of reasoning has been put forward which essentially turns this observation around [15]: given that the solution of a truncated flow depends on the regulator, it should be possible to identify specific ones which "optimize" the physical content of a given approximation. Optimized regulators stabilize the flow and lead to a faster convergence of expansions, such that the main physical 
information is almost exclusively contained within a few leading terms, and higher order contributions remain small [18].

In Ref. [15], we have derived a simple and generic optimization criterion for ERG flows, based only on the full inverse propagator at vanishing field. Given the set of possible IR regulators, the criterion allows to distinguish the quality of regulators in the sense outlined above. In the present paper we study a specific "optimized" regulator for both bosonic and fermionic degrees of freedom. To be more explicit, we introduce the ERG flow for the effective action $[4-6]$. For bosonic fields $\phi$, it is given by

$$
\partial_{t} \Gamma_{k}[\phi]=\frac{1}{2} \operatorname{Tr}\left(\frac{\delta^{2} \Gamma_{k}}{\delta \phi(q) \delta \phi(-q)}+R_{k}\right)^{-1} \partial_{t} R_{k} .
$$

Here, the trace denotes a sum over all loop momenta and indices, and $t=\ln k$ is the logarithmic scale parameter. The flow has a simple one-loop structure. The Wilsonian "integrating-out" is achieved by the infrared regulator $R_{k}$. It regulates the propagator for small momenta, while the insertion $\partial_{t} R_{k}$ cuts off the large-momentum contributions. In total, only a small momentum window about $q^{2} \approx k^{2}$ contributes to the flow. Apart from a few constraints displayed later, the function $R_{k}$ can be chosen at will. A "good" choice for the regulator function is at the root of reliable physical predictions, and we consider, for the bosonic fields, the optimized regulator

$$
R_{k}^{\mathrm{opt}}\left(q^{2}\right)=Z_{k}\left(k^{2}-q^{2}\right) \Theta\left(k^{2}-q^{2}\right),
$$

where $Z_{k}$ is an appropriately defined wave function renormalization. This regulator is particularly simple: for loop momenta $q^{2}>k^{2}$ it vanishes identically and the effective propagator appearing in the flow equation is not modified; for loop-momenta $q^{2}<k^{2}$ it acts like a momentumdependent mass term in such a way that the inverse effective propagator $\sim q^{2}+R_{k}\left(q^{2}\right)$ becomes a momentum independent constant. In consequence, the effective infra-red propagator no longer distinguishes between the different modes with $q^{2}<k^{2}$.

Optimized flows based on Eq. (1.2) derive from a generic optimization criterion [15], and have a number of remarkable properties. The optimized flow leads to the fastest decoupling of heavy modes, in accordance with the decoupling theorem [19]. In the limit $k \rightarrow 0$, optimized flows smoothly approach a convex effective action, owing to a simple analytic pole of the flow $[15,18]$. At non-vanishing temperature, the optimized flow factorizes: the contributions from thermal and quantum fluctuations are disentangled, unlike for generic flows. A similar factorization of the flow holds to second order in the derivative expansion for field-independent wave function renormalizations, and a partial factorization is found for the general case. Finally, the optimized flow has a very simple analytic structure. This facilitates their study and is helpful for both analytical or numerical considerations. All these properties lead to a stabilization of the flow and an improved convergence towards the physical theory. Analogous results for fermionic flows are discussed as well.
We also study this question within an $\mathrm{RG}$ formalism based on a proper-time regularization of the operator trace for the one-loop effective action [20], which we call the "proper-time renormalization group" (PTRG) for short. In contrast to the ERG, the PTRG has no path integral derivation, which makes the conceptual reasoning more difficult [21]. Still, owing to the close similarity to the ERG at leading order in the derivative expansion, it is possible to identify the analog of Eq. (1.2) for the PTRG.

The format of the paper is as follows. We introduce the physical ideas behind the generic optimization condition. Explicit realizations for bosonic and fermionic degrees of freedom are introduced as well (Sec. II). The main characteristics of optimized flows are discussed to leading order in the derivative expansion, and contrasted with those of generic flows (Sec. III). We then turn to the discussion of quantum field theories at finite temperature. We show that optimized thermal flows factorize on the level of the flow equation, unlike generic flows. A simple physical explanation for the factorization is provided (Sec. IV). Next, we consider the extension to higher orders in the derivative expansion. The cases of field dependent or independent wave-function renormalizations are both discussed, and a similar factorization for optimized flows is established (Sec. V). Finally, we provide the corresponding optimized proper-time cutoff for the PTRG (Sec. VI). Because of the qualitative difference between the topics studied, we discuss our findings separately at the end of the corresponding sections. We close with a summary and an outlook (Sec. VII). Three Appendixes contain technical details and explicit expressions for optimized flows.

\section{OPTIMIZATION}

In this section, we discuss a generic optimization criterion for ERG flows for Euclidean quantum field theories. In particular, we provide a simple and explicit optimized regulator for both bosonic and fermionic flows. Prior to this, we have to review a few basic properties of IR regulator functions, which are at the root of the subsequent considerations.

\section{A. Regulators}

The flow equation (1.1) is defined through the infrared regulator functions $R_{k}\left(q^{2}\right)$ and $R_{F, k}\left(q^{2}\right)$, respectively [4-6]. These operators depend on an infrared scale $k$, which induces a scale dependence. When written in terms of the scaledependent effective action $\Gamma_{k}$, the scale dependence is given precisely by the flow equation (1.1). The right-hand side of Eq. (1.1) contains the full inverse propagators and the trace denotes a sum over all indices and integration over all momenta.

The regulator scheme (RS) functions can be chosen at will, however, within some basic restrictions. These restrictions ensure that the flow equation is well-defined, thereby interpolating between an initial action in the UV and the full quantum effective action in the IR. More specifically, it is required that 


$$
\lim _{q^{2} / k^{2} \rightarrow 0} R_{k}\left(q^{2}\right)>0 .
$$

This ensures that the effective propagator at vanishing field remains finite in the infrared limit $q^{2} \rightarrow 0$, and no infrared divergences are encountered in the presence of massless modes. This property makes $R_{k}$ an infrared regulator. If the limit (2.1) is finite, we call the corresponding regulator masslike. The second requirement is the vanishing of $R_{k}$ in the infrared,

$$
\lim _{k^{2} / q^{2} \rightarrow 0} R_{k}\left(q^{2}\right) \rightarrow 0 .
$$

This guarantees that the regulator function is removed in the physical limit, where the scale-dependent effective action $\Gamma_{k}$ reduces to the quantum effective action $\Gamma=\lim _{k \rightarrow 0} \Gamma_{k}$. The third condition to be met is

$$
\lim _{k \rightarrow \Lambda} R_{k}\left(q^{2}\right) \rightarrow \infty .
$$

This way it is ensured that $\Gamma_{k}$ approaches the microscopic action $S=\lim _{k \rightarrow \Lambda} \Gamma_{k}$ in the UV limit $k \rightarrow \Lambda$. In the rest of the paper, we set $\Lambda=\infty$ for the UV scale, although our main line of reasoning can be applied for finite $\Lambda$ as well. With this choice, the regulator function depends only on $q^{2}$ and $k^{2}$, and it is convenient to introduce a dimensionless function $r\left(q^{2} / k^{2}\right)$ as

$$
R_{k}\left(q^{2}\right)=Z_{k} q^{2} r\left(q^{2} / k^{2}\right)
$$

with $Z_{k}$ an appropriate wave function renormalization (cf. Sec. V); $Z_{k} \equiv 1$ to leading order in the derivative expansion. Owing to the general conditions imposed on the regulator, the function $r(y)$ ranges between $0 \leqslant r(y) \leqslant \infty$.

Another condition concerns the proper normalization of the regulator. The normalization fixes the scale at which the IR regulator becomes effective. Let us employ the condition

$$
R_{k}\left(q^{2}=c_{B} k^{2}\right)=Z_{k} c_{B} k^{2}
$$

for bosons (a similar condition holds for fermions, see below) and $c_{B}>0 .{ }^{1}$ The normalization translates into the condition $r\left(c_{B}\right)=1$. Two different choices for $c_{B}$ can always be mapped onto each other through a rescaling of the IR scale $k$. Hence, a proper normalization is only of relevance for a comparison of different regulators (as done in Ref. [15]), or for theories containing different bosonic and/or fermionic degrees of freedom, where the relative normalization of the regulators can become important.

\section{B. Optimization criterion}

Here, we discuss an optimization criterion for ERG flows, which ensures that flows like Eq. (1.1) and approximations to it have good convergence and stability properties. Following Ref. [15] (see also Ref. [18]), we first provide the general

\footnotetext{
${ }^{1}$ In Ref. [15] the convention $c_{B}=1$ has been used.
}

criterion for optimized choices of RS functions. Then, more specifically, we apply this idea to bosonic and fermionic theories with standard kinetic terms.

The physical information of the flow equation (1.1) is contained in the full effective inverse propagator, which is given by

$$
\frac{\delta^{2} \Gamma_{k}[\phi]}{\delta \phi(q) \delta \phi(-q)}+R_{k}\left(q^{2}\right)
$$

Notice that Eq. (2.6) depends both on the fields and on the RS function. The ERG flow is well-defined as long as the full inverse propagator displays a gap,

$$
\min _{q^{2} \geqslant 0}\left(\left.\frac{\delta^{2} \Gamma_{k}[\phi]}{\delta \phi(q) \delta \phi(-q)}\right|_{\phi=\phi_{0}}+R_{k}\left(q^{2}\right)\right)=C k^{2}>0 .
$$

The functional derivative is evaluated at a properly chosen expansion point $\phi_{0}$. The existence of the gap $C>0$ implies an IR regularization. Furthermore, the gap is a prerequisite for the ERG formalism. Otherwise, Eq. (1.1) becomes singular at points where the full inverse effective propagator develops zero modes. ${ }^{2}$ The size of the gap $C$ in Eq. (2.7) depends both on the RS function and on dimensionless parameters like $\phi_{0}^{2} / k^{2}$ or mass ratios, specific to the particular theory studied.

A natural optimization criterion based on Eq. (2.7) consists of maximizing the gap $C$ over the space of all possible RS functions. Optimized RS functions are those for which the maximum of $C$ is attained. The optimization ensures that the momentum-dependent kernel of the ERG flow is the most regular. Therefore we expect that optimized flows are much more stable against approximations and show better convergence properties.

The optimization condition as formulated above is, essentially, only sensitive to the momentum dependence of the full inverse propagator. Dropping momentum-independent terms on the left-hand side of Eq. (2.7) changes the number $C$ accordingly, but leaves the explicit dependence on $R_{k}\left(q^{2}\right)$ unchanged. Therefore, the optimization leads to the same set of optimized RS functions as long as the implicit dependence of $\Gamma_{k}^{(2)}[\phi] \equiv \delta^{2} \Gamma_{k}[\phi] / \delta \phi(q) \delta \phi(-q)$ on the RS function remains negligible. For this reason, the optimization condition of Refs. [15,18] is based only on the momentumdependent terms of Eq. (2.6).

From now on, we concentrate on a standard kinetic term. The effect of a field-dependent wave function renormalization can be taken into account as well (see Sec. V below). We expand the full inverse propagator as $Z_{k}\left[q^{2}+Z_{k}^{-1} R_{k}\left(q^{2}\right)\right.$ $+\ldots]$ about the regularized kinetic term. Finally, dropping the momentum-independent terms transforms Eq. (2.7) into

\footnotetext{
${ }^{2}$ The case $C=0$ indicates that a saddle point expansion about $\phi_{0}$ is not applicable. Those points $\phi_{0}$ in field space with $C=0$ correspond to an instability. The problem can be solved by choosing a more appropriate expansion point such that $C>0$. For related literature, see Ref. [22].
} 


$$
\min _{q^{2} \geqslant 0}\left[q^{2}+Z_{k}^{-1} R_{k}\left(q^{2}\right)\right]=C k^{2}>0 .
$$

A far reaching consequence of the infrared regulator in Eq. (2.8) is the presence of a gap for all $k>0$, which follows trivially from Eq. (2.1). The decisive difference between Eq. (2.7) and Eq. (2.8) is that the size of the gap $C>0$ in Eq. (2.8) depends only on the particular choice for the RS, but not on the specific theory. Rewriting Eq. (2.8) in dimensions of $k$ leads to

$$
P^{2}(y) \equiv q^{2} / k^{2}+R_{k}\left(q^{2}\right) /\left(Z_{k} k^{2}\right)=y[1+r(y)],
$$

where $y \equiv q^{2} / k^{2}$. Expressed in terms of Eq. (2.9), the size of the gap is given by

$$
C=\min _{y \geqslant 0} P^{2}(y) .
$$

Any RS function is now characterized by the associated gap $C$. The size of the gap can be made arbitrarily small. Effectively, this corresponds to removing the IR regulator in the first place. However, for fixed normalization $c_{B}$, it cannot be made arbitrarily large, $C<\infty$. Hence, the natural optimization condition, which is the requirement to maximize the gap, becomes

$$
C_{\mathrm{opt}}=\underset{(\mathrm{RS})}{\max }\left(\min _{y \geqslant 0} P^{2}(y)\right) .
$$

A few comments are in order. The maximum in Eq. (2.11) is taken over the (infinite-dimensional) space of all possible RS functions. The number $C_{\mathrm{opt}}$ is uniquely determined and reads $C_{\text {opt }}=2 c_{B}$, where $c_{B}$ is the normalization of bosonic regulators. From now on, we refer to Eq. (2.11) as an "optimization condition," and all RS functions for which $C=C_{\mathrm{opt}}$ are called solutions to the optimization condition. The space of solutions to the optimization condition is infinitedimensional. Notice also that the condition to minimize the gap is not an extremization linked to the regulator, because it corresponds to removing the IR regularization. In Ref. [15], a variety of different solutions have been found, and some examples are given in Fig. 1 below.

In order to obtain Eq. (2.8), we have assumed a standard kinetic term for the fields. Therefore, the resulting optimization condition Eq. (2.11) is independent of the specific theory. Once the momentum-dependent part of $\Gamma_{k}^{(2)}$ depends on the fields, the corresponding optimization condition based on the momentum-dependent part of Eq. (2.7) is sensitive to the specific theory. Within a derivative expansion, this happens starting from the second order (cf. the discussion in Sec. V).

The optimization condition has a number of interpretations in more physical terms (cf. Refs. [15,18]). It has been shown that the radius of convergence for amplitude expansions is given by $C$. Therefore the optimization condition improves their convergence. Furthermore, it leads to a smooth approach towards a convex effective potential in the IR limit $k \rightarrow 0$. It has also been shown that it improves the convergence of the derivative expansion [18]. Finally, it is worth emphasizing that the optimization criterion is a rather

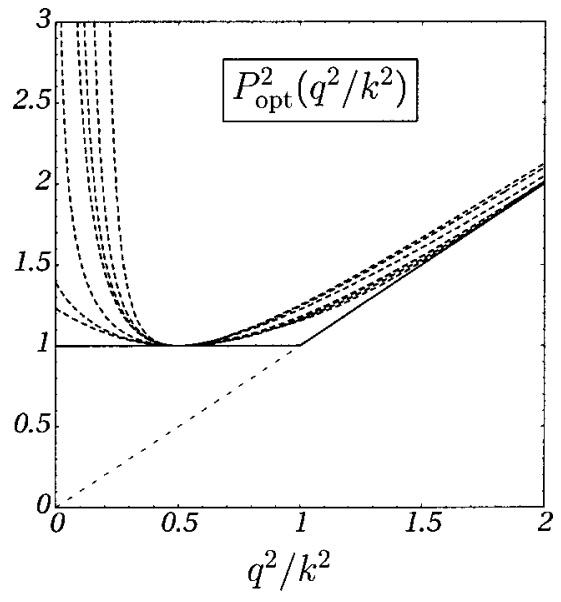

FIG. 1. Optimized inverse propagators $P_{\mathrm{opt}}^{2}$ for different regulators, normalized as $r\left(\frac{1}{2}\right)=1$. The regulator Eq. (2.18) is given by the full line. The thin dashed line corresponds to $r=0$. All other dashed lines, given for comparison, correspond to the different optimized regulators of Fig. 3 in Ref. [15].

mild condition: all regulator functions are described by at most countably infinitely many parameters, because $R_{k}$ is at least square integrable. Of these, only one parameter is fixed by the optimization criterion.

We now turn to the discussion of fermionic degrees of freedom $\psi$ and $\bar{\psi}[23,24]$. The flow equation is given by

$$
\partial_{t} \Gamma_{k}[\psi, \bar{\psi}]=-\operatorname{Tr}\left(\frac{\delta^{2} \Gamma_{k}}{\delta \psi(q) \delta \bar{\psi}(-q)}+R_{F, k}\right)^{-1} \partial_{t} R_{F, k} .
$$

As usual, the trace sums over all loop momenta and indices. The constraints on the function $R_{F, k}$ are similar to those on $R_{k}$ [24]. Following Ref. [24], we choose the regulator proportional to \& and introduce

$$
R_{F, k}(q)=Z_{F, k} \phi r_{F}\left(q^{2} / k^{2}\right) .
$$

We choose the normalization as

$$
R_{F, k}^{2}\left(q^{2}=c_{F} k^{2}\right)=c_{F} k^{2} .
$$

This translates into the condition $r_{F}\left(c_{F}\right)=1$. It has been shown that the fermionic analog of the function Eq. (2.9) is given by [24]

$$
P_{F}^{2}(y)=y\left[1+r_{F}(y)\right]^{2} .
$$

Therefore, we can define the fermionic gap as

$$
C_{F}=\min _{y \geqslant 0} P_{F}^{2}(y),
$$

and the corresponding optimization condition reads

$$
C_{F, \text { opt }}=\underset{(\mathrm{RS})}{\max }\left(\min _{\geqslant 0} P_{F}^{2}(y)\right) .
$$


The optimized fermionic gap is uniquely determined through the normalization $c_{F}$ as $C_{F \text { opt }}=4 c_{F}$. Conceptually, the fermionic case is treated in the same way as the bosonic case. The sole difference stems from the fact that the bosonic kinetic term contains two derivatives, while the fermionic kinetic term contains only one. Therefore, the functions (2.9) and (2.15) entering the optimization condition are different.

\section{Derivation of optimized bosonic and fermionic regulators}

A lot of effort has been made in order to provide explicit regulators which lead to sufficiently simple and analytic ERG flows. For example, the sharp cutoff provides a simple explicit flow to leading order in the derivative expansion. For this reason, it is one of the most intensively studied flows in the field (cf. Refs. $[1,2,25,6,26,27]$ ). Other attempts have been made based on power-like regulators $R_{k} \sim q^{2}\left(k^{2} / q^{2}\right)^{b}$ for $b=1$ and $b=2$ [28], or variants of a mass-term regulator $R_{k} \sim k^{2} \Theta\left(k^{2}-q^{2}\right)$. These regulators are still sufficiently simple from an algebraic point of view, and lead to reasonably simple flows. ${ }^{3}$ However, in the absence of an underlying "guiding principle" it was not obvious how to make progress given the plethora of possible regulators, and in particular, how to distinguish the "quality" of the corresponding flows.

Here, in turn, we take full advantage of the existence of a guideline provided by the optimization criterion. We propose a regulator which (i) solves the optimization criterion, (ii) is based on an additional stability criterion for approximate flows, and (iii) leads to simple explicit expressions for the corresponding flows. The heuristic derivation runs as follows. The space of regulators which solve the optimization criterion is still infinite dimensional. Let us seek a "simple" solution to Eq. (2.11). The simplest one corresponds to an inverse propagator which is flat, i.e., momentumindependent, $P^{2} \equiv C_{\mathrm{opt}}$. Take $C_{\mathrm{opt}}=1$. This immediately implies, using Eqs. (2.4) and (2.9), that $R_{k}\left(q^{2}\right)=k^{2}-q^{2}$. Our naive ansatz is consistent with Eqs. (2.1) and (2.3), but not with the main requirement Eq. (2.2) for small $k^{2}<q^{2}$. In order to fulfill Eq. (2.2), the regulator has to be cut off above some loop momenta. Therefore, a natural proposal for the bosonic case consists in taking

$$
R_{k}^{\mathrm{opt}}\left(q^{2}\right)=\left(k^{2}-q^{2}\right) \Theta\left(k^{2}-q^{2}\right) .
$$

The ultraviolet modes $q^{2}>k^{2}$ are not touched by this regulator because Eq. (2.18) vanishes identically for $q^{2}>k^{2}$. In turn, for all modes with $q^{2} \leqslant k^{2}$ the regulator acts as a momentum-dependent mass term $\sim\left(k^{2}-q^{2}\right)$ with the infrared limit $\sim k^{2}$ for vanishing momenta. It is a masslike regulator. By construction, the inverse propagator at vanishing field Eq. (2.6) becomes momentum independent for all $q^{2}$ $\leqslant k^{2}$ (see Fig. 1). It is this property which is responsible for the main characteristics of the regulator: all infrared momentum modes below the scale $k$ are treated in the same way since the effective inverse propagator no longer distinguishes between them.

\footnotetext{
${ }^{3}$ Of these, only the power-like regulator with $b=2$ solves the optimization condition Eq. (2.11).
}

The dimensionless regulator function $r(y)$ is defined in Eq. (2.4). With the choice made in Eq. (2.18) it follows that

$$
r_{\mathrm{opt}}(y)=\left(\frac{1}{y}-1\right) \Theta(1-y) .
$$

The regulator function is normalized with $c_{B}=\frac{1}{2}$. Such a normalization can always be achieved. Other normalizations are considered at the end of Sec. III.

In Fig. 1, we have displayed the effective inverse propagator at vanishing field for different optimized regulators. The full line represents Eq. (2.18), and the thin dashed line corresponds to $R=0$ (no regulator). The set of dashed lines corresponds to the optimized regulators discussed in Fig. 3 of Ref. [15]. Here, they have been given for comparison. Notice that all curves cross in the normalization point $r\left(c_{B}\right)=1$. All optimized propagators display the same gap $C_{\text {opt }}=2 c_{B}$, but differ essentially in the curvature around their minima.

The fermionic analog of Eq. (2.18) is derived in essentially the same way. Starting with Eq. (2.15), imposing $P_{F}^{2}$ $\equiv 1$ for small momenta, and the general conditions (2.1), (2.2) and (2.3), we finally end up with

$$
R_{F, k}^{\mathrm{opt}}(q)=q\left(\sqrt{\frac{k^{2}}{q^{2}}}-1\right) \Theta\left(k^{2}-q^{2}\right),
$$

normalized with $c_{F}=\frac{1}{4}$. In terms of a dimensionless function $r_{F}\left(q^{2} / k^{2}\right)$, Eq. (2.20) becomes

$$
r_{F, \mathrm{opt}}(y)=\left(\frac{1}{\sqrt{y}}-1\right) \Theta(1-y)
$$

and $r_{F}\left(\frac{1}{4}\right)=1$. The non-analyticity of Eq. (2.21) is a direct consequence of $R_{F, k}$ having only one mass dimension. We shall see below that it is of no harm to the computation of fermionic flows because Eq. (2.21) enters only in specific combinations such that the non-analyticity disappears.

\section{DERIVATIVE EXPANSION}

The flow equation (1.1) is a functional differential equation, which, from a technical point of view, is equivalent to infinitely many coupled partial differential equations for the couplings parametrizing the effective action $\Gamma_{k}$. A number of different systematic approximation procedures for flows are known. In this section, we consider flows to leading order in the derivative expansion, based on expanding the operators of the effective action according to the number of derivatives [29]. This leads to a closed set of coupled partial differential equations for the coefficient functions. We discuss the main structure of optimized flows and contrast it with generic ones.

\section{A. Specific flows}

In order to make our subsequent reasoning more transparent, it is useful to have an explicit example at hand. To that end, we consider an $O(N)$-symmetric real scalar field theory 
in $d$ dimensions, the linear sigma model. To leading order in the derivative expansion we make the ansatz [30]

$$
\begin{aligned}
\Gamma_{k}= & \int d^{d} x\left(U_{k}(\bar{\rho})+\frac{1}{2} Z_{k}(\bar{\rho}) \partial_{\mu} \phi^{a} \partial_{\mu} \phi_{a}+\frac{1}{4} Y_{k}(\bar{\rho}) \partial_{\mu} \bar{\rho} \partial_{\mu} \bar{\rho}\right. \\
& \left.+\mathcal{O}\left(\partial^{4}\right)\right)
\end{aligned}
$$

for the effective action, with $\bar{\rho}=\frac{1}{2} \phi^{a} \phi_{a}$. For $N \neq 1$, there are two independent wave function factors $Z_{k}$ and $Y_{k}$ beyond leading order in this expansion (cf. Sec. V). To leading order in the derivative expansion, the flow equation (1.1) reduces to a flow for the effective potential, $\partial_{t} U_{k}$. The main physical applications concern the non-trivial Wilson-Fisher fixed point in $d=3$ and the computation of related universal quantities.

Inserting the ansatz (3.1) into the basic flow equation, and using $Z \equiv Y \equiv 1$, yields [30]

$$
\begin{aligned}
\partial_{t} U_{k}= & \frac{1}{2} \int \frac{d^{d} q}{(2 \pi)^{d}}\left(\frac{(N-1) \partial_{t} R_{k}\left(q^{2}\right)}{q^{2}+R_{k}\left(q^{2}\right)+U_{k}^{\prime}(\bar{\rho})}\right. \\
& \left.+\frac{\partial_{t} R_{k}\left(q^{2}\right)}{q^{2}+R_{k}\left(q^{2}\right)+U_{k}^{\prime}(\bar{\rho})+2 \bar{\rho} U_{k}^{\prime \prime}(\bar{\rho})}\right) .
\end{aligned}
$$

It is a second order non-linear partial differential equation. One easily recognizes the contributions from the $N-1$ "Goldstone" modes and the "radial" mode. A similar flow equation has been obtained for the wave function renormalizations $Z_{k}$ and $Y_{k}$ [30]. The momentum integration is regularized in the UV, owing to the regulator term $\partial_{t} R_{k}\left(q^{2}\right)$ in the numerator, and in the IR due to $R_{k}\left(q^{2}\right)$ in the denominator.

\section{B. Generic flows}

For convenience we perform the angular part of the momentum integration and rewrite the right-hand side of Eq. (3.2) in terms of so-called threshold functions [30] as

$$
\begin{aligned}
\partial_{t} U_{k}(\bar{\rho})= & 2 v_{d}(N-1) k^{d} l_{0}^{d}\left(\frac{U_{k}^{\prime}(\bar{\rho})}{k^{2}}\right) \\
& +2 v_{d} k^{d} l_{0}^{d}\left(\frac{U_{k}^{\prime}(\bar{\rho})+2 \bar{\rho} U_{k}^{\prime \prime}(\bar{\rho})}{k^{2}}\right) .
\end{aligned}
$$

The constants $v_{d}$ are given by

$$
v_{d}^{-1}=2^{d+1} \pi^{d / 2} \Gamma\left(\frac{d}{2}\right),
$$

and the functions $l_{n}^{d}(\omega)$ are defined as

$$
l_{n}^{d}(\omega)=\left(\delta_{n, 0}+n\right) \int_{0}^{\infty} d y y^{(d / 2)-1} \frac{-y^{2} r^{\prime}(y)}{\left[P^{2}(y)+w\right]^{n+1}} .
$$

While the flow (3.3) is specific for the theory defined by Eq. (3.1), the functions (3.5) are not. These functions describe the generic structure of the flow to leading order in the derivative expansion. The flows for different indices $n>0$ are related by

$$
\partial_{\omega} l_{n}^{d}(\omega)=-\left(n+\delta_{n, 0}\right) l_{n+1}^{d}(\omega) .
$$

Therefore, it suffices to study the flows $l_{0}^{d}(\omega)$.

The fermionic analog of the flow (3.5) is [24]

$$
l_{F, n}^{d}(\omega)=\left(\delta_{n, 0}+n\right) \int_{0}^{\infty} d y y^{d / 2} \frac{-2 y\left(1+r_{F}\right) r_{F}^{\prime}}{\left[P_{F}^{2}(y)+w\right]^{n+1}}
$$

and Eq. (3.6) holds equally for Eq. (3.7). Notice the additional factor $1-r_{F}$ in the integrand, which arises due to the Dirac structure of Eq. (2.20). We have used the normalization condition $r_{F}\left(\frac{1}{4}\right)=1$.

It is evident that the characteristics of the flow, determined by the choice of $R_{k}$, are entirely encoded within the functions Eqs. (3.5) and (3.7) (or similar functions to higher order in the derivative expansion). For a generic regulator, these are complicated functions of the fields, which can be computed explicitly only for very specific choices for the regulator.

Two properties of generic flows given in terms of $l_{0}^{d}(\omega)$ and $l_{F, 0}^{d}(\omega)$ are worth mentioning. First of all, from their very definition and the constraints imposed on the regulator function, we conclude that any function (3.5) for $n=0$ decays at most as $1 / \omega$ for $\omega \rightarrow \infty$ [30]. Therefore, they describe the decoupling of "heavy" modes from the flow, which is a manifestation of the decoupling theorem [19]. Secondly, all flows have a pole in $C+\omega$, where $C$ denotes the gap. Both the analytical structure and the strength of the pole depend on the regulator. From the general requirements for regulators, and the explicit form of Eq. (3.5), it follows that the pole for $n=0$ cannot be stronger than a simple analytical pole $\sim 1 /(C+\omega)$. The pole of threshold functions has important physical implications. It determines the approach to a convex effective potential for theories within a phase of spontaneous symmetry breaking $[31,8]$.

\section{Optimized flows}

Now we turn our attention to the optimized regulators introduced in Eqs. (2.18) and (2.20). The evaluation of Eq. (3.5) is particularly simple because the $\Theta$-function cuts off the momentum integration. Using Eq. (2.19), Eq. (3.5) reduces to two terms,

$$
\begin{aligned}
l_{0}^{d}(\omega)= & \frac{1}{1+w} \int_{0}^{1} d y y^{(d / 2)-1} \\
& +\int_{0}^{\infty} d y y^{(d / 2)-1} \frac{(y-1) \delta(1-y)}{1-y \Theta(1-y)+w} .
\end{aligned}
$$

In the first term, the momentum integration is cut off above $y \leqslant 1$. The function $P^{2}(y)$ remains a constant in this momentum regime, which allowed to move the $\omega$-dependent term in 
front of the momentum integration. The integrand of the second term contains products of distributions. Since the integrand is proportional to $\sim(1-y) \delta(1-y)$ the second term vanishes identically, independently of the specific implementation for the $\Theta$-function. The remaining momentum integration of the first term becomes trivial and gives

$$
l_{0}^{d}(\omega)=\frac{2}{d} \frac{1}{1+\omega} .
$$

We used the normalization $r\left(\frac{1}{2}\right)=1$ and hence $P^{2}=1$ for $y$ $\leqslant 1$.

For fermionic flows (3.7) and the regulator (2.20), we find

$$
\begin{aligned}
l_{F, 0}^{d}(\omega)= & \frac{1}{1+\omega} \int_{0}^{1} d y y^{(d / 2)-1}+2 \int_{0}^{\infty} d y y^{(d / 2)+1} \\
& \times \frac{\left[1+\left(\frac{1}{\sqrt{y}}-1\right) \Theta(1-y)\right]\left(\frac{1}{\sqrt{y}}-1\right) \delta(1-y)}{[\sqrt{y}+(1-\sqrt{y}) \Theta(1-y)]^{2}+w} .
\end{aligned}
$$

The first term has a restricted momentum integration due to the cutoff provided by the $\Theta$-function. The second term is more involved, and the integrand even contains products of distributions. Notice, however, that it contains the factor $\sim[(1 / \sqrt{y})-1] \delta(1-y)$ which is proportional to $\sim(y$ -1) $\delta(1-y)$. Therefore, the second term vanishes identically and independent of the parametrization of the distributions and their products. The evaluation of the first term gives finally

$$
l_{F, 0}^{d}(\omega)=\frac{2}{d} \frac{1}{1+\omega},
$$

and is identical to the bosonic flow.

\section{Discussion}

The flows described by the functions (3.9) and (3.11) have the simplest asymptotic structure for $\omega \rightarrow \infty$. This implies that heavy modes decouple "the fastest" from the flow for optimized regulators. For comparison, the sharp cutoff leads only to a logarithmic decoupling $\sim \ln \omega$. Also, the decoupling does not depend on the particular theory studied (i.e. the dimension), unlike the case for polynomial regulators $R_{k} \sim q^{2}\left(k^{2} / q^{2}\right)^{b}$. Furthermore, the flow described by the functions (3.9) and (3.11) has the simplest and strongest pole structure for $C+\omega \rightarrow 0^{+}$. The pole is a simple analytic one, which is not the case for generic regulator functions. An immediate implication of this structure is that the optimized flows (3.9) and (3.11) lead to a logarithmically smooth approach towards a convex effective potential. This is very different from the sharp cutoff case, where the approach is only exponential [8]. A detailed presentation of these results is given elsewhere.

For completeness we quote the results for the flows (3.9) and (3.11) for arbitrary normalization. While the normaliza- tion is of no relevance for a theory containing only bosonic or fermionic degrees of freedom, their relative normalization can become important for theories containing bosons and fermions. The normalization conditions (2.5) and (2.14) correspond to $r\left(c_{B}\right)=1$ and $r_{F}\left(c_{F}\right)=1$, which can always be imposed because the functions $r(y)$ and $r_{F}(y)$ range between $0 \leqslant r, r_{F} \leqslant \infty$. The optimized gaps are $C_{\mathrm{opt}}=2 c_{B}$ and $C_{F \text { opt }}=4 c_{F}$. For arbitrary $c_{B}$ the flow Eq. (3.9) is obtained as

$$
l_{0}^{d}(\omega)=\frac{2}{d} \frac{\left(2 c_{B}\right)^{(d / 2)+1}}{2 c_{B}+\omega} .
$$

In the fermionic case we find

$$
l_{F, 0}^{d}(\omega)=\frac{2}{d} \frac{\left(4 c_{F}\right)^{(d / 2)+1}}{4 c_{F}+\omega}
$$

for the rescaled analog of Eq. (3.11).

Finally, we note that flows $l_{0}^{d}(\omega) \sim 1 /(1+\omega)$ have been used earlier in the literature [32-34], however without the explicit knowledge of the corresponding regulator function. These trial functions are sufficiently simple to allow for analytical considerations. The motivation for their use was based on the observation that the generic threshold function Eq. (3.5) decays at most as $\omega^{-1}$ for large $\omega$. This suggested that a regulator may exist which leads to $l_{0}^{d}(\omega)=A_{d}(C+\omega)^{-1}$. Let us show how the normalization $A_{d}$ can be derived from consistency arguments. We use the universal relation $l_{n}^{2 n}(0)$ $=1$ [30], which holds for $d=2 n$ dimensions, to identify the prefactor as $A_{2 n}=(1 / n) C^{n+1}$. The analytic continuation to arbitrary dimensions leads finally to our results (3.12) and (3.13). This reasoning shows that the ansatz $l_{0}^{d}(\omega)$ $=(2 / d) C^{d / 2+1}(C+\omega)^{-1}$ is self-consistent. However, we rush to add that these consistency arguments are necessary conditions, but not sufficient ones: only the explicit form of the regulator — as given by Eqs. (2.18) and (2.20) - finally justifies the few earlier computations. In addition, Eqs. (2.18) and (2.20) are explicitly required for the computation of the flow at finite temperature (see Sec. IV) or to higher order (see Sec. V).

\section{THERMAL FLUCTUATIONS}

In this section we apply our reasoning in the context of a quantum field theory coupled to a heat bath at temperature $T$, and to leading order in the derivative expansion. We show that optimized flows, as opposed to generic ones, disentangle the different contributions related to thermal and quantum fluctuations, respectively. These properties are realized, on the level of the flow equation, in terms of an important $f a c$ torization. This leads to better convergence properties of the flow itself. Approximate solutions of the flow correspond to better approximations of the physical theory.

\section{A. Imaginary time formalism}

To be explicit, we consider a bosonic or fermionic field theory at thermal equilibrium at the temperature $T$ within the 
Matsubara formalism. This implies that periodic (antiperiodic) boundary conditions for the bosonic (fermionic) fields are employed. As a consequence, the $q_{0}$ integration in the flow equation (1.1) is replaced by a sum over Matsubara modes $m=0, \pm 1, \pm 2, \ldots$ The trace in Eq. (1.1) contains a momentum integration, which is then substituted as

$$
\int \frac{d^{d} q}{(2 \pi)^{d}} \rightarrow T \sum_{m} \int \frac{d^{d-1} q}{(2 \pi)^{d-1}} .
$$

In the integrand of Eq. (1.1) the $q_{0}$ variable is replaced by

$$
q_{0} \rightarrow 2 \pi c_{m} T
$$

where

$$
\begin{aligned}
& c_{m}=m \quad \text { for bosons } \\
& c_{m}=m+\frac{1}{2} \quad \text { for fermions. }
\end{aligned}
$$

It is also useful to introduce the variable

$$
\tau=2 \pi T / k
$$

for the following considerations. The replacement (4.2) implies that functions $l_{n}^{d}(\omega)$ turn into temperature dependent functions $l_{n}^{d}(\omega, \tau)$. We show that this function factorizes for the regulators (2.18) and (2.20).

\section{B. Dimensional reduction and fermion decoupling}

Let us review a few basic facts known for generic flows at finite temperature within the imaginary time formalism $[30,35,24,36]$.

Bosonic fields within the Matsubara formalism display the phenomenon of dimensional reduction at high temperature. This means that for $T$ large enough all non-vanishing Matsubara modes are suppressed due to effective masses $\sim m T$ for the Matsubara modes with $m \neq 0$. Only the $m=0$ mode survives in this limit, leading to an effective theory in $(d-1)$-dimensions. For a generic bosonic regulator, the finite temperature flow is given as

$$
\begin{aligned}
l_{0}^{d}(\omega, \tau)= & \frac{v_{d-1}}{v_{d}} \frac{\tau}{2 \pi} \sum_{m} \int_{0}^{\infty} d y y^{[(d-1) / 2]-1} \\
& \times \frac{-\left(y+c_{m}^{2} \tau^{2}\right)^{2} r^{\prime}\left(y+c_{m}^{2} \tau^{2}\right)}{P^{2}\left(y+c_{m}^{2} \tau^{2}\right)+\omega} .
\end{aligned}
$$

The function $P^{2}$ is defined in Eq. (2.9). The asymptotic regime where only the $m=0$ Matsubara mode contributes is reached for $\tau \rightarrow \infty$. From Eqs. (4.6) and (3.6), we deduce

$$
l_{n}^{d}(\omega, \tau \rightarrow \infty)=\frac{v_{d-1}}{v_{d}} \frac{T}{k} l_{n}^{d-1}(\omega) .
$$

On the other hand, the limit $\tau \rightarrow 0$ eventually switches on all higher order Matsubara modes. It is straightforward to verify that

$$
l_{n}^{d}(\omega, \tau \rightarrow 0)=l_{n}^{d}(\omega) .
$$

The asymptotic limits for $T \rightarrow \infty$, Eq. (4.7), and $T \rightarrow 0$, Eq. (4.8), display dimensional reduction for bosons as a function of temperature for generic regulator function [35].

Fermions at finite temperature within the Masubara formalism can be treated in essentially the same way. However, they happen to have no $m=0$ mode as antiperiodic boundary conditions have to be used on the $q_{0}$-integration. Hence, fermions do not display dimension reduction. Rather, they decouple completely from the RG flow once the smallest Matsubara mode is larger than the scale $k$. These properties can be read off from the temperature-dependent flow. For a generic fermionic regulator, the flow $l_{F, 0}^{d}(\omega)$ at finite temperature is defined as

$$
\begin{aligned}
l_{F, 0}^{d}(\omega, \tau) & \\
= & \frac{v_{d-1}}{v_{d}} \frac{\tau}{2 \pi} \sum_{m} \int_{0}^{\infty} d y y^{[(d-1) / 2]-1} \\
& \times \frac{-2\left(y+c_{m}^{2} \tau^{2}\right)^{2} r_{F}^{\prime}\left(y+c_{m}^{2} \tau^{2}\right)\left[1+r_{F}\left(y+c_{m}^{2} \tau^{2}\right)\right]}{P_{F}^{2}\left(y+c_{m}^{2} \tau^{2}\right)+\omega} .
\end{aligned}
$$

The function $P_{F}^{2}$ is given in Eq. (2.15). The asymptotic regime where the fermions decouple completely is reached for $\tau \rightarrow \infty$. From Eq. (4.9), we deduce that

$$
l_{F, n}^{d}(\omega, \tau \rightarrow \infty)=0 .
$$

Again, the limit $\tau \rightarrow 0$ eventually switches on all higher order Matsubara modes such that

$$
l_{F, n}^{d}(\omega, \tau \rightarrow 0)=l_{F, n}^{d}(\omega) .
$$

The asymptotic limits (4.10) and (4.11) describe the decoupling of fermions in the high temperature limit for arbitrary dimension and generic regulator function.

\section{Optimized thermal flows and factorization}

We now turn to the optimized regulators (2.18) and (2.20). For this case, the flow (4.6) can be computed explicitly. Inserting Eq. (2.18) into Eq. (4.6), and following a reasoning analogous to the one after Eq. (3.8), we find

$$
l_{n}^{d}(\omega, \tau)=B_{d}(\tau) l_{n}^{d}(\omega)
$$

with the temperature dependent function

$$
B_{d}(\tau)=\frac{d}{d-1} \frac{v_{d-1}}{v_{d}} \frac{\tau}{2 \pi} \sum_{m}\left(1-c_{m}^{2} \tau^{2}\right)^{(d-1) / 2} \Theta\left(1-c_{m}^{2} \tau^{2}\right) .
$$

Notice that the temperature effects have factorized. This implies that temperature cuts off all amplitudes $\omega$ in the same manner. This is not the case for a generic regulator.

Let us discuss the thermal threshold factor $B_{d}(\tau)$. In Fig. 2 the thermal threshold factor $B_{d}(\tau)$ is displayed for $d$ 


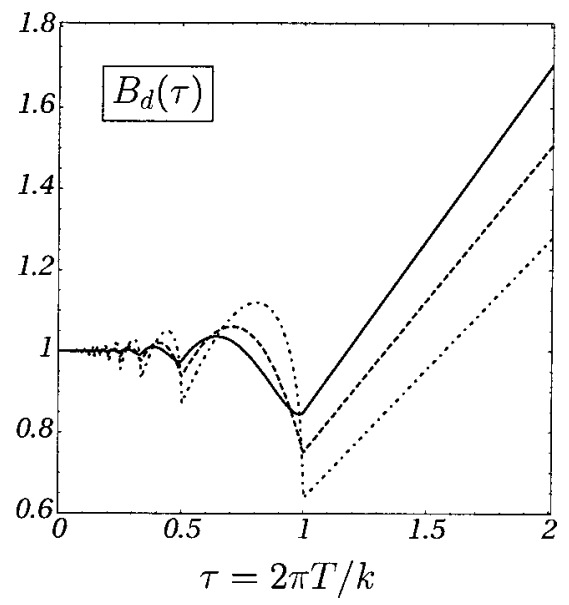

FIG. 2. Dimensional reduction for bosons, described by the bosonic thermal function $B_{d}(\tau)$ defined in Eq. (4.13). $d=4$ : full line, $d=3$ : dashed line, $d=2$ : dashed-dotted line.

$=2,3$ and 4 dimensions. Every single Matsubara mode contributes to Eq. (4.13) proportional to

$$
\sim \tau\left(1-c_{m}^{2} \tau^{2}\right)^{(d-1) / 2} \Theta\left(1-c_{m}^{2} \tau^{2}\right) .
$$

The $\Theta$-function is a remnant of the regulator (2.19) and cuts the $m$ th Matsubara mode off as soon as $k<c_{m} T / 2 \pi$. The factor $\tau$ stems from the $q_{0}$-integration and the factor (1 $\left.-c_{m}^{2} \tau^{2}\right)^{(d-1) / 2}$ from the $d-1$ dimensional integration over spatial loop momenta $|\mathbf{q}|$. These functions vanish outside the interval $0 \leqslant \tau \leqslant 1 / c_{m}$. At the upper end they behave like $\left(1 / c_{m}-\tau\right)^{(d-1) / 2}$ and vanish linearly with $\tau$ at the lower end. This structure explains the spikes observed in Fig. 2, which are located precisely at the points $\tau=1 / c_{m}$ and due to the decoupling of the $\pm c_{m}$ th Matsubara modes. Indeed, for $\tau$ $>1$ only the $m=0$ Matsubara mode yields a contribution to $B_{d}(\tau)$ in Eq. (4.13). The asymptotic regime where only the $m=0$ Matsubara mode contributes is reached already for $\tau$ $>1$ with $B_{d}(\tau \geqslant 1)=\tau d v_{d-1} / 2 \pi v_{d}(d-1)$, or

$$
l_{n}^{d}(\omega, \tau \geqslant 1)=\frac{v_{d-1}}{v_{d}} \frac{T}{k} l_{n}^{d-1}(\omega) .
$$

Notice the difference from Eq. (4.7). Decreasing $\tau$ below $\tau$ $=1 / c_{m}$ eventually switches on the $\pm c_{m}$ Matsubara modes. For $\tau$ close to the points $1 / c_{m}$, the term (4.14) increases as $\left(1 / c_{m}-\tau\right)^{(d-1) / 2}$ for decreasing $\tau$. This power law explains why the spikes are more pronounced in lower dimensions. ${ }^{4}$ In the limit $\tau \rightarrow 0$ it is straightforward to verify that $B_{d}(\tau$ $\rightarrow 0) \rightarrow 1$ which implies

$$
l_{n}^{d}(\omega, \tau \rightarrow 0)=l_{n}^{d}(\omega) .
$$

This asymptotic limit is the same as Eq. (4.8).

\footnotetext{
${ }^{4}$ To higher order in the derivative expansion, the spikes are smoothed out for non-trivial wave function renormalization, cf. Sec. V C.
}

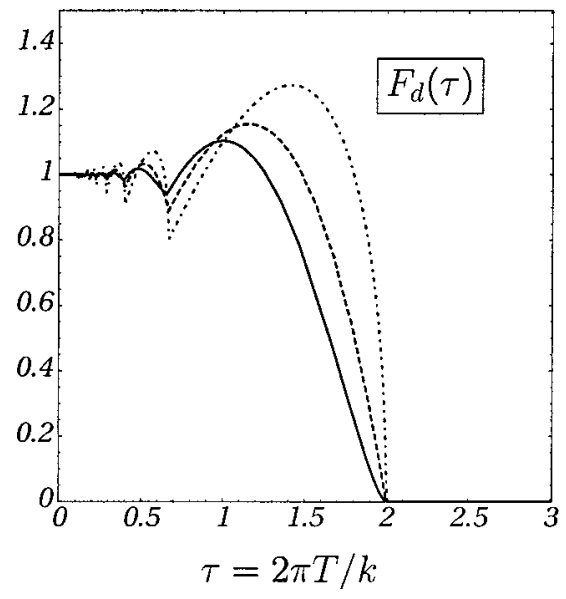

FIG. 3. Fermion decoupling at finite temperature described by the fermionic thermal function $F_{d}(\tau)$ given by Eq. (4.18). $d=4$ : full line, $d=3$ : dashed line, $d=2$ : dashed-dotted line.

Turning to the optimal fermionic regulator (2.21), the momentum integration in Eq. (4.9) can be performed explicitly to give

$$
l_{F, n}^{d}(\omega, \tau)=F_{d}(\tau) l_{F, n}^{d}(\omega)
$$

As in the bosonic case, the temperature effects factorize from the threshold effects. The fermionic thermal threshold factor $F_{d}(\tau)$ is given by

$$
F_{d}(\tau)=\frac{d}{d-1} \frac{v_{d-1}}{v_{d}} \frac{\tau}{2 \pi} \sum_{m}\left(1-c_{m}^{2} \tau^{2}\right)^{(d-1) / 2} \Theta\left(1-c_{m}^{2} \tau^{2}\right) .
$$

Equation (4.18) is identical to its bosonic counterpart (4.13) except for the Matsubara sum which runs over $c_{m}= \pm \frac{1}{2}$, $\pm \frac{3}{2}, \ldots$ in Eq. (4.18). In Fig. 3 we have displayed the function $F_{d}(\tau)$ for $d=2,3$ and 4 dimensions. Again, the spikes have the same origin as in the bosonic case and the same reasoning applies. The high temperature limit at which the fermions decouple completely, is already reached for $k$ $\leqslant \pi T$,

$$
l_{F, n}^{d}(\omega, \tau \geqslant 2)=0 .
$$

Notice the important difference from Eq. (4.10), where the decoupling of fermions is only asymptotic. The limit $\tau \rightarrow 0$ is equivalent to Eq. (4.11).

\section{Discussion}

The optimized regulators (2.18) and (2.20) correctly describe dimensional reduction and fermion decoupling. In addition, they lead to a thermal factorization of the flow as observed in Eqs. (4.12) and (4.17). From a physical point of view, this fact is easily understood. The imaginary time formalism compactifies the time direction and the temperature modifies the temporal momentum modes of the fields. The corresponding Matsubara mode, when compared to the infrared scale $k$, leads to a thermal decoupling. To leading order in the derivative expansion, the optimized regulator makes the 
temperature blind for the quantum fluctuations. It cannot distinguish between amplitudes of constant fields. In turn, the quantum fluctuations are sensitive to the field amplitudes, which are responsible for the mass decoupling, similar to the case for vanishing temperature. Clearly, these two effects have different physical origins. As a consequence, it is natural to employ a regulator which reflects this factorization on the level of the flow equation.

For a generic regulator function, the flows (4.6) and (4.9) are complicated functions of both the temperature and the field amplitudes. They reflect dimensional reduction and fermion decoupling. Typically, however, they do not factorize. This simply means that a generic ERG flow entangles thermal and quantum fluctuations even to leading order in the derivative expansion. This is a direct consequence of the regulator term, whose coupling to the different operators in the effective action leads to a field-dependent thermal decoupling of the different modes on the level of the flow equation. This entanglement is of no relevance if the flow can be solved exactly. In turn, for an approximate solution of the flow, the factorization on the level of the flow equation is most helpful. It avoids a mixing of thermal and quantum fluctuations in a regime where they can be disentangled, thereby minimizing possible artifacts due to the specific regulator function. As a consequence, the flow itself is stabilized, and expansions of the flow show much better convergence behavior towards the physical theory. More generally, it is expected that this line of reasoning applies for generic optimized regulators.

Finally, the factorization is very helpful for numerical solutions of flow equations. In the generic case, one twoparameter function has to be fitted in order to describe the flows (4.6) or (4.9). In turn, only two one-parameter functions are needed once they factorize as in Eqs. (4.12) and (4.17). This simplification is substantial, and even more so, because the functions (4.12) and (4.17) have a very simple analytical form.

\section{DERIVATIVE EXPANSION TO SECOND ORDER}

In this section, we apply the optimized regulator to higher order in the derivative expansion. We first discuss the general structure of the equations. Furthermore, we show that a simple factorization of the flow takes place for fieldindependent wave function renormalization. The physical origin of the factorization is discussed, and its realization on the level of the flow equation leads, as in the thermal case, to better convergence properties of the flow and the derivative expansion. For technical details on the computations, we defer to the Appendixes.

\section{A. Wave-function renormalization}

In the preceding sections we have restricted the discussion to the leading order in a derivative expansion. This implied the vanishing of the anomalous dimensions $\eta_{\phi}=\eta_{\psi}=0$ or $Z_{\phi} \equiv Z_{\psi} \equiv 1$. To higher order in the derivative expansion the multiplicative renormalization of the fields has to be taken into account, according to

$$
\begin{gathered}
\phi \rightarrow Z_{\phi, k}^{1 / 2} \phi \\
\psi \rightarrow Z_{\psi, k}^{1 / 2} \psi .
\end{gathered}
$$

Once higher derivative terms are included in the ansatz for the effective action, additional flow equations for the corresponding coefficient functions like $Z_{\phi, k}\left(q^{2}, \bar{\rho}\right)$ and $Z_{\psi, k}\left(q^{2}, \bar{\rho}\right)$ have to be studied. The wave function renormalizations are functions of the scale parameter $k$ and can depend as well on momenta $q^{2}$ or on the mean fields $\bar{\rho}$. To second order in the derivative expansion the wave function renormalization is evaluated at a particular momentum scale $q^{2}=k_{0}^{2}$ which fixes the renormalization conditions. Typical choices for $k_{0}$ are $k_{0}=0$ and $k_{0}=k$.

The most important new ingredient at this order is the scale- and field-dependence of the wave function renormalizations. In the example defined through Eq. (3.1), these are given by the functions $Z_{k}$ and $Y_{k}$. Here, the function $Z_{k}$ is responsible for the renormalization of the $N-1$ "Goldstone" modes, which differs from $\widetilde{Z}_{k}=Z_{k}+\bar{\rho} Y_{k}$ for the "radial" mode. The fact that different wave function renormalizations appear to second order in the derivative expansion (depending on the theory considered) is of no relevance for the following discussion of the flows. The parametric dependence of the flow on either $Z_{k}$ or $\widetilde{Z}_{k}$ is the same.

Let us introduce an additional function $z_{k}(\rho)$ as

$$
Z_{k}(\bar{\rho})=Z_{k}\left(\bar{\rho}_{0}\right) z_{k}(\bar{\rho})
$$

We have factored out a constant term $Z_{k}\left(\bar{\rho}_{0}\right)$ chosen at an arbitrary reference point. We have chosen the reference point $\bar{\rho}=\bar{\rho}_{0}$ which fixes the renormalization of the fields for all momenta. Typical choices are either $\rho_{0}=0$, or $\rho_{0}=$ the minimum of the scale-dependent potential. The split (5.3) allows to separate the non-trivial field-dependence, contained in $z_{k}(\bar{\rho})$, from an overall renormalization contained in $Z_{k}\left(\bar{\rho}_{0}\right)$. The factor $z_{k}$ is normalized as $z_{k}\left(\bar{\rho}_{0}\right)=1$.

In order to provide a simple form for the flow it is useful to introduce the field-independent factor $Z_{k}\left(\bar{\rho}_{0}\right)$ into the regulator function,

$$
\begin{gathered}
R_{k} \rightarrow Z_{\phi, k} R_{k} \\
R_{F, k} \rightarrow Z_{\psi, k} R_{F, k} .
\end{gathered}
$$

The flow equation, when written in terms of renormalized variables (5.1) and (5.2), receives additional contributions proportional to the anomalous dimensions

$$
\begin{aligned}
& \eta_{\phi}=-\partial_{t} \ln Z_{\phi, k}, \\
& \eta_{\psi}=-\partial_{t} \ln Z_{\psi, k},
\end{aligned}
$$

because the derivative $\partial_{t} R_{k}$ in the flow equation now acts also on the explicit scale-dependence contained in $Z_{k}$. 


\section{B. Generic flows to second order}

To second order in the derivative expansion the flow has turned into a function of the field amplitudes $\omega$, the anomalous dimension $\eta$ and the field dependent function $z$. The corresponding bosonic flow is defined as

$$
l_{0}^{d}(\omega, z, \eta)=\int_{0}^{\infty} d y y^{(d / 2)-1} \frac{-y^{2} r^{\prime}(y)-\frac{1}{2} \eta y r(y)}{y[z+r(y)]+\omega} .
$$

Notice that the pole structure of the flow is changed, owing to the function $z(\rho)$. The effective inverse propagator becomes a function of the fields:

$$
P^{2}(\rho, y)=y[z(\rho)+r(y)] .
$$

The location of the pole of Eq. (5.8) at $-\omega=C(\rho)$ $=\min _{y \geqslant 0} P^{2}(\rho, y)$ has turned into a function of the fields. For the optimized regulator (2.19) the pole is located at $C$ $=\min \{1, z\}$. Compared to the leading order in the derivative expansion, the pole structure is modified once $z \neq 1$.

For the fermionic case, the flows are given as

$$
l_{F, 0}^{d}\left(\omega, z_{F}, \eta_{\psi}\right)=\int_{0}^{\infty} d y y^{d / 2} \frac{-\left(2 y r_{F}^{\prime}+\eta_{\psi} r_{F}\right)\left(z_{F}+r_{F}\right)}{y\left[z_{F}+r_{F}(y)\right]^{2}+\omega} .
$$

The pole structure changed as well, as follows from

$$
P_{F}^{2}(\rho, y)=y\left[z_{F}(\rho)+r_{F}(y)\right]^{2} .
$$

The location of the pole of Eq. (5.8) at $-\omega=C_{F}(\rho)$ $=\min _{y \geqslant 0} P_{F}^{2}(\rho, y)$ has turned into a function of the fields. For the regulator (2.21) the pole is located at $C_{F}=\min \left\{1, z_{F}\right\}$. The pole structure is modified compared to the leading order in the derivative expansion once $z_{F} \neq 1$.

\section{Optimized flows to second order}

We now turn to the optimized flows and discuss their structure at second order in the derivative expansion. We refer to the Appendixes for all technical details.

In the case of a generic wave-function renormalization where $z_{k}(\bar{\rho})$ is a non-trivial function of the fields, the function $l_{0}^{d}(\omega, z, \eta)$ as defined in Eq. (5.8) can be evaluated explicitly for the regulator (2.18). The structure of the flow is as follows. Consider the denominator of Eq. (5.8), given by $y(z+r)+\omega$. It can be rewritten as $1+\omega+y(z-1)+[y(1$ $+r)-1]$. The last term in brackets vanishes for the optimized regulator (2.18) because the integration is restricted to $y \leqslant 1$. The remaining term can be written as the product ( 1 $+\omega)\{1-[(1-z) /(1+\omega)] y\}$. Notice also that the numerator of Eq. (5.8) depends neither on $z$ nor on $\omega$. These observations lead to the following conclusions. First, and apart from an overall $\omega$-dependence $\sim(1+\omega)^{-1}$, the optimized flow depends on $z$ and $\omega$ only through the variable

$$
\xi \equiv \frac{1-z}{1+\omega} .
$$

Second, the optimized flow factorizes into a leading order term (3.9) and a remaining factor $B_{d}(\xi, \eta)$,

$$
l_{0}^{d}(\omega, \xi, \eta)=l_{0}^{d}(\omega) B_{d}(\xi, \eta)
$$

because the denominator of Eq. (5.8) contains a momentumindependent factor $(1+\omega)$. Here we have introduced $l_{0}^{d}(\omega, \xi(\omega, z), \eta) \equiv l_{0}^{d}(\omega, z, \eta)$. It it interesting to see that the structure of the optimized flow is still quite simple. An integral representation of $B_{d}(\xi, \eta)$ is given in Eq. (B3). [For all $\xi<1, B_{d}(\xi, \eta)$ can be expressed in terms of hypergeometric functions, cf. Eqs. (B4), (B5) and (B6); closed expressions of Eq. (5.13) for $d=4,3$ and 2 dimensions are given in Eqs. (B7), (B8) and (B9), respectively.] For $|\xi|<1$ the function $B_{d}(\xi, \eta)$ can be Taylor-expanded in arbitrary dimensions, to wit

$$
\begin{aligned}
B_{d}(\xi, \eta) & =\sum_{n=0}^{\infty} \frac{\xi^{n}}{1+\frac{2 n}{d}}\left(1-\frac{\eta}{d+2+2 n}\right) \\
& =1-\frac{\eta}{d+2}+\frac{\xi}{1+\frac{2}{d}}+\mathcal{O}\left(\xi^{2}, \xi \eta\right)
\end{aligned}
$$

The series representation (5.14) is best suited for the flow as long as $|\xi|$ remains small. This corresponds to either the limit of a field-independent wave function renormalization $z(\rho)$ $\equiv 1$, or, for any $z$, to the limit of large amplitudes $\omega$. From Eq. (5.14), we obtain for Eq. (5.13) to zeroth order in $\xi$

$$
l_{0}^{d}(\omega, \xi, \eta)=l_{0}^{d}(\omega)\left(1-\frac{\eta}{d+2}\right)+\mathcal{O}\left(\frac{\xi}{1+\omega}, \frac{\xi \eta}{1+\omega}\right) .
$$

We note that the $\eta$-dependent correction in Eq. (5.15) has the same functional dependence on the amplitude $\omega$ as Eq. (3.9). Stated differently, the optimized regulator leads to a simple factorization in both the decoupling limit $\omega \gg 1$ and for the case of a field-independent anomalous dimension $\xi \equiv 0$.

For completeness we give also the result for the bosonic flow at finite temperature. The corresponding flow $l_{0}^{d}(\omega, \xi, \tau, \eta)$ still factorizes as

$$
l_{0}^{d}(\omega, \xi, \tau, \eta)=B_{d}(\xi, \tau, \eta) l_{0}^{d}(\omega) .
$$

This is the generalization of Eq. (4.12) to second order in the derivative expansion. We only have to replace the function $B_{d}(\xi, \eta)$ by its temperature-dependent counterpart $B_{d}(\xi, \tau, \eta)[\mathrm{cf}$. Eq. (A5)]. It is straightforward, if tedious, to establish explicitly that the function $B_{d}(\xi, \tau, \eta)$ represents dimensional reduction in precisely the same way as $B_{d}(\tau)$ $\equiv B_{d}(\xi=0, \tau, \eta=0)$. Let us consider the most interesting case, which is the leading order in $\xi \ll 1$. In this limit, Eq. (5.16) reads

$$
B_{d}(\xi, \tau, \eta)=B_{d}(\tau, \eta)+\mathcal{O}(\xi, \xi \eta) .
$$

The function $B_{d}(\tau, \eta)$ can be expressed as 


$$
\begin{aligned}
B_{d}(\tau, \eta)= & B_{d}(\tau)-\eta \frac{d}{d^{2}-1} \frac{v_{d-1}}{v_{d}} \frac{\tau}{2 \pi} \\
& \times \sum_{m}\left(1-c_{m}^{2} \tau^{2}\right)^{(d+1) / 2} \Theta\left(1-c_{m}^{2} \tau^{2}\right) .
\end{aligned}
$$

The function $B_{d}(\tau)$, Eq. (4.13), has been discussed in Sec. IV. The new ingredient, beyond leading order, is given by the corrections $\sim \eta$ in Eq. (5.18). Every single Matsubara mode contributes as

$$
\sim \tau\left(1-c_{m}^{2} \tau^{2}\right)^{(d+1) / 2} \Theta\left(1-c_{m}^{2} \tau^{2}\right)
$$

Compared to the leading order contributions (4.14), we notice that Eq. (5.19) follows from Eq. (4.14) for $d \rightarrow d+2$. The reason is very simple. In the flow equation, the anomalous dimension is proportional to a term containing an additional factor $\sim q^{2}$, which effectively increases the momentum measure by two dimensions. This has an immediate consequence. The thermal decoupling in Eq. (5.18) proportional to $\sim \eta$ is much smoother than the leading order decoupling, simply because the spikes are less pronounced the higher the dimension. Therefore, the spikes observed in Fig. 2 are smoothed out once $\eta$ (and $\xi$ ) are non-vanishing.

In the opposite regime where $|1-z| /(1+w) \gg 1$, only a few leading terms of the series (5.14) have to be retained. This limit is of relevance close to the pole region of the flows $\omega \rightarrow-1$, or in the region of large $z \gg 1$. From the explicitly resummed expressions (B7), (B8) and (B9), we conclude that a factorization as

$$
l_{0}^{d}(\omega, z, \eta)=f_{d}(\omega, z)\left(1-\frac{\eta}{d}\right)
$$

holds true, and $f_{4}(z)=(z-1)^{-1}, \quad f_{3}(z)=2 f_{4}(z)$ and $f_{2}(\omega, z)=f_{4}(z) \ln ((z+\omega) /(1+\omega))$.

It is not surprising that a similar structure is found for fermionic flows. The correction term due to the substitution Eq. (5.4) simplifies Eq. (5.10) to

$$
\begin{aligned}
& l_{F, 0}^{d}\left(\omega, z_{F}, \eta_{\psi}\right) \\
& \quad=\int_{0}^{1} d y y^{d / 2-1} \frac{\left[1+\sqrt{y}\left(z_{F}-1\right)\right]\left[1-\eta_{\psi}(1-\sqrt{y})\right]}{\left[1+\sqrt{y}\left(z_{F}-1\right)\right]^{2}+w} .
\end{aligned}
$$

Equation (5.21) factorizes as

$$
l_{F, 0}^{d}\left(\omega, z_{F}, \eta_{\psi}\right)=l_{F, 0}^{d}(\omega) F_{d}\left(\omega, z_{F}, \eta_{\psi}\right) .
$$

The function $F_{d}(\omega, z, \eta)$ can be expressed in terms of hypergeometric functions. At finite temperature, and for $z=1$ and $\eta=0$, it reduces to Eq. (4.18). Here, we are only interested in the structure of the flow for a nearly field-independent wave function renormalization, $z \approx 1$, or for the decoupling limit. We find

$$
\begin{aligned}
F_{d}\left(\omega, z_{F}, \eta_{\psi}\right)= & 1-\frac{\eta_{\psi}}{d+1}+\frac{d\left(z_{F}-1\right)}{d+1}\left(1-\frac{2}{1+\omega}\right) \\
& \times\left(1-\frac{\eta_{\psi}}{d+2}\right)+\cdots .
\end{aligned}
$$

The two leading terms in Eq. (5.23) show that also fermionic flows factorize for field-independent wave function renormalization.

\section{Discussion}

The structure of the flow has increased to second order in the derivative expansion. Let us discuss first the case of a field-independent wave function renormalization $z \equiv 1$. The corresponding flows (5.15) and (5.23) for the optimized regulators factorize, similar to the thermal case to leading order in the derivative expansion. Physically speaking, this structure can be made plausible as follows. The flow, when written in terms of the renormalized fields - and under the assumption that the renormalization is momentum- and fieldindependent-depends, in addition to the fields, only on the anomalous dimension. The anomalous dimension is field independent, and, as a consequence, unable to distinguish between fields of different amplitudes contained in $\omega$, which parametrize the quantum fluctuations. Therefore, it is natural that the flow factorizes the contributions induced through $\eta$ from those induced by the amplitudes $\omega$. The disentanglement is realized by the optimized regulators. ${ }^{5}$

In turn, a generic flow does not reflect this factorization. Rather, it leads to an entanglement between the renormalization of the effective potential induced by the infrared regulator, and the renormalization parametrized by a fieldindependent anomalous dimension. This is immediately evident from the observation that the $\eta$-dependent and the $\eta$-independent contributions to the flow of the effective potential have different functional forms as functions of the fields. At this level, the entanglement is due to the regulator, which modifies the coupling among all operators of the effective action. As mentioned in the thermal case, the entanglement is of no importance for the full solution to the flow. In turn, the factorization is very useful for approximate solutions. It leads to more stable flows because irrelevant couplings, entirely due to the regulator, are removed. The same reasoning as given at the end of the previous section applies.

For the thermal bosonic flow (5.16), we notice that the dependence on the anomalous dimension enters the thermal factor $B_{d}(\tau, \eta)$. In particular, the thermal corrections do not factorize from those due to a field-independent anomalous dimension. This structure can be understood as follows. The wave function renormalization enters the momentum trace as a multiplicative renormalization proportional to the kinetic

\footnotetext{
${ }^{5}$ From the definition of Eqs. (5.8) and (5.10) it follows that all homogeneous regulators with $r(y) \sim y r^{\prime}(y)$ [or $r_{F}(y) \sim y r_{F}^{\prime}(y)$, respectively] factorize the anomalous dimension from the fielddependent part of the flow.
} 
term $q^{2}$. At finite temperature within the imaginary time formalism, the spatial and the temporal loop momenta are treated in an unequal way. Hence, thermal fluctuations couple in a non-trivial manner to the anomalous dimension of the fields. This implies that the temperature-dependent factor itself is modified due to the anomalous dimension, which provides the physical reason why no factorization of the temperature effects from the anomalous dimension are expected in the first place.

For the case of a field-dependent wave-function renormalization, a simple factorization similar to Eq. (5.15) is not expected, simply because the wave function renormalization is a function of the fields. Hence, the wave function renormalization can distinguish different field amplitudes, in contrast to the field-independent case. However, two observations are still worth mentioning. First of all, we observe a partial factorization, which is evident from Eqs. (5.13) and (5.16). This structure is based on the fact that the $z$-dependence enters only through the variable (5.12), as opposed to the generic case. For $|1-z| /(1+w) \ll 1$, only a few leading terms have to be retained from the explicit series (5.14). It follows that each power of $|1-z| /(1+w)$ is renormalized proportional to the anomalous dimension and an order-dependent numerical coefficient. Secondly, the limit for $|1-z| /(1+w) \gg 1$ again allows for a simple factorization, as follows from Eq. (5.20). Here, the wave function renormalization can no longer distinguish field amplitudes, allowing for this simple structure.

A final comment concerns the numerical prefactors $\sim \eta$ as found in Eqs. (5.15) and (5.23). We emphasize that the coupling of the anomalous dimensions to the effective potential is, apart from the field dependence, dimensionally suppressed-by factors $1 /(d+2)$ for bosons and $1 /(d+1)$ for fermions-as opposed to the leading order contributions. This additional suppression is noteworthy because the convergence of the derivative expansion is controlled by small anomalous dimensions of the fields. Here, we have just shown that an expansion performed with an optimized regulator leads to an additional dimensional suppression of the back-coupling of the anomalous dimension to the effective potential. A more detailed discussion of this observation will be given elsewhere.

\section{PROPER-TIME REGULARIZATION}

In this section we leave aside the conceptual framework of the ERG based on a momentum-scale regularization and address flows based on an operator cutoff regularization. Our aim is to provide the analog of the optimized regulator (2.18) within the proper-time regularization method. For a more detailed comparison with the exact renormalization group, we refer the reader to Ref. [21].

A simple flow has been derived from a one-loop expression for the effective action which is UV and IR regularized using a Schwinger proper-time representation of the operator trace [37], amended by a regulator function $f_{k}^{(d)}(\Lambda, s)$ within the proper-time integral [38]. The flow with respect to the infrared scale parameter $k$ follows from a 1-loop improvement as [20]

$$
\partial_{t} \Gamma_{k}=-\frac{1}{2} \int_{0}^{\infty} \frac{d s}{s}\left[\partial_{t} f_{k}^{(d)}(\Lambda, s)\right] \operatorname{Tr} \exp \left(-s \Gamma_{k}^{(2)}\right) .
$$

We refer to this flow as the "proper-time renormalization group" (PTRG). It describes the partial resummation of perturbative diagrams. The proper-time regulator function plays the role of the momentum regulator $R_{k}$ within the ERG. The flow (6.1) is governed by the IR scale $k$. Following Ref. [20], we introduce a dimensionless function $f(x)$ as $f_{k}^{(d)}(\Lambda, s)$ $=f\left(\Lambda^{2} s\right)-f\left(k^{2} s\right)$ and require $f(x \rightarrow \infty)=1$ and $f(x \rightarrow 0)$ $=0$. This ensures that the usual Schwinger proper time representation is reached in the UV limit.

We are not aware of a simple and generic optimization criterion, analogous to Eq. (2.11), which derives from within the PTRG formalism. Furthermore, the flow (6.1) has no path integral derivation, which makes a conceptual reasoning much more difficult. However, it is still possible to show that a function $f_{\text {opt }}(x)$ exists which is equivalent to the optimized ERG regulator (2.18) to the leading order in the derivative expansion.

To that end, we apply Eq. (6.1) to an $N$-component real scalar theory in $d$ dimensions and to leading order in the derivative expansion. Using the ansatz (3.1) the flow for the effective potential $U_{k}(\bar{\rho})$ with $\bar{\rho}=\frac{1}{2} \phi_{a} \phi_{a}$ becomes

$$
\begin{aligned}
\partial_{t} U_{k}(\bar{\rho})= & \frac{1}{2}(4 \pi)^{-d / 2} \int_{0}^{\infty} \frac{d s}{s^{1+d / 2}} \partial_{t} f_{k}^{(d)}(\Lambda, s) \\
& \times\left[e^{-s\left[U_{k}^{\prime}(\bar{\rho})+2 \bar{\rho} U_{k}^{\prime \prime}(\bar{\rho})\right]}+(N-1) e^{-s U_{k}^{\prime}(\bar{\rho})}\right] .
\end{aligned}
$$

This flow is identical in form to the ERG flow (3.3), if we replace the ERG flow in Eq. (3.3) by the proper-time flow

$$
l_{0}^{d}(\omega)=\frac{1}{2} \Gamma\left(\frac{d}{2}\right) \int_{0}^{\infty} d x x^{-1-(d / 2)}\left[\partial_{t} f(x)\right] \exp (-x \omega) .
$$

Here, the integration variable is $x=k^{2} s$ and stems from the proper-time integration, in contrast to Eq. (3.5), where $y$ $=q^{2} / k^{2}$ stems from the momentum trace. Now, consider a specific class of proper-time regulator functions:

$$
f(x)=\frac{\Gamma(m, x)}{\Gamma(m)}, \quad \partial_{t} f(x)=\frac{2 x^{m} e^{-x}}{\Gamma(m)} .
$$

We have introduced a free parameter $m$ describing different regulators, and the incomplete $\Gamma$-function $\Gamma(m, x)$ $=\int_{0}^{x} d y y^{m-1} e^{-y}$. This yields the simple expression

$$
l_{0}^{d}(\omega)=\frac{\Gamma\left(m-\frac{d}{2}\right) \Gamma\left(\frac{d}{2}\right)}{\Gamma(m)}(1+\omega)^{(d / 2)-m}
$$

which agrees with Eq. (3.9) for $m=1+(d / 2)$, or 


$$
f^{\mathrm{opt}}(x)=\frac{\Gamma\left(\frac{d}{2}+1, x\right)}{\Gamma\left(\frac{d}{2}+1\right)}, \quad \partial_{t} f^{\mathrm{opt}}(x)=\frac{2 x^{1+d / 2} e^{-x}}{\Gamma\left(\frac{d}{2}+1\right)} .
$$

The optimized proper-time regulator (6.6) corresponds to the optimized regulator (2.18) within the ERG approach. Hence, it is possible to identify an optimal regulator function for proper-time flows, owing to their close similarity to the ERG to leading order in the derivative expansion.

\section{CONCLUSIONS AND OUTLOOK}

This study was motivated by two observations. First, an application of the ERG to realistic physical problems is bound to certain approximations. Second, approximate solutions of flow equations depend spuriously on the infrared regulator. Combining these observations, it became obvious that an understanding of the spurious scheme dependence is mandatory in order to provide predictive power for approximate solutions. Previously, we showed that the gap of the full inverse propagator controls convergence properties of approximate solutions [15]. It has also been shown, based on the computation of critical exponents for the Ising universality class, that the convergence of the derivative expansion is controlled by the gap [18]. These observations lead to the conclusion that the freedom in the choice for the IR regulator can be used to maximize the physical information contained within a given approximation or truncation.

An interpretation of the interplay between the RS function on one side, and convergence of approximate flows on the other, is as follows. The IR regulator-by regulating the flow-modifies the interactions at intermediate scales $k \neq 0$ among all operators of the theory. Eventually, these cancel out for the integrated full flow, but not for approximated ones. Hence, changing the RS function for an approximated flow modifies some remaining RS dependent terms which cannot be cancelled due to the missing contributions from neglected operators. Therefore, a "fine-tuning" of the RS function allows to partly incorporate higher-order effects within the lower orders of a given approximation. This corresponds to an optimization.

The present derivation of optimized ERG flows had two ingredients. First, we made use of a generic optimization criterion for bosonic and fermionic fields [15], which states that the gap of the full inverse propagator, as a function of momenta, should be as large as possible. This way, the ERG flow is the least singular, and approximations to such flows are expected to be much more stable, leading to improved convergence of expansions. Second, we added the specific requirement that the effective inverse propagator be momentum-independent in the IR region (cf. Fig. 1).

We have studied specific optimized ERG flows for bosonic or fermionic theories up to second order in the derivative expansion and at vanishing and non-vanishing temperature. Their specific properties have been discussed in detail at the end of the corresponding sections. Here, let us only mention perhaps the most surprising property of opti- mized flows, which is the disentanglement of thermal and quantum fluctuations to leading order in the derivative expansion. A similar factorization occurs for field-independent wave function renormalizations.

More generally, optimized ERG flows owe their main properties to the "flatness" of the effective inverse propagator, which extends over the entire momentum region $q^{2}$ $\leqslant k^{2}$ for the specific regulator studied here. Other regulators can lead to similar factorization and convergence properties. Prime candidates are given by solutions to the optimization criterion: as is evident from Fig. 1, they automatically lead to flat effective propagators-at least within a small region about the minimum of the effective inverse propagator. If this region extends over the domain where the flow equation receives its main contributions, we expect to find equally good flows.

An important conclusion is that the optimization ideas discussed here should be useful for high-precision computations based on this formalism. Increasing the precision normally implies a full computation at the following order of the expansion. Here, we argued that the physical results can be improved already within a fixed order of the expansion. Immediate applications of optimized flows concern the computation of universal critical exponents for $N$-component scalar theories in three dimensions, or the study of convex effective potentials within a phase with spontaneous symmetry breaking. On the conceptual side, it is possible to show that the optimization criterion can be interpreted as a natural minimum sensitivity condition, somewhat similar to the principle of minimum sensitivity as employed within perturbative QCD. We will leave a detailed discussion of these results to a future publication [18].

Our analysis can be extended in a number of directions. For gauge theories, modified Ward or BRST identities ensure the gauge invariance of physical Green functions [39], and the optimization criterion is compatible with such additional constraints. This optimization can also be implemented for field theories at finite temperature within the real-time formalism [36]. While our present analysis is based on the derivative expansion, it seems worthwhile to study optimizations for other systematic expansions like expansions in powers of the fields. Finally, it would be interesting to see how these ideas apply to flows at finite density or to Hamiltonian flows [40].

\section{ACKNOWLEDGMENT}

This work has been supported by EC Contract no. HPMFCT-1999-00404.

\section{APPENDIX A: FLOWS TO SECOND ORDER IN THE DERIVATIVE EXPANSION}

In this appendix, we derive explicit expressions for the optimized flow for the effective potentials to second order in the derivative expansion at both vanishing and non-vanishing temperature.

Our starting point is the flow for the effective potential to second order in the derivative expansion. We consider the 
bosonic flow at finite temperature. All information about the flow is parametrized by

$$
\begin{aligned}
l_{0}^{d}(\omega, z, \tau, \eta)= & \frac{v_{d-1}}{v_{d}} \frac{\tau}{2 \pi} \sum_{n} \int_{0}^{1-c_{n}^{2} \tau^{2}} d y y^{(d-3) / 2} \\
& \times \frac{\left[1-\frac{1}{2} \eta\left(1-y-c_{n}^{2} \tau^{2}\right)\right] \Theta\left(1-c_{n}^{2} \tau^{2}\right)}{(z-1)\left(y+c_{n}^{2} \tau^{2}\right)+1+\omega} .
\end{aligned}
$$

Here, $\omega(\rho)$ is the field variable, $z(\rho)$ a field dependent wave function renormalization, $\eta$ the anomalous dimension, $\tau$ $=2 \pi T / k$ the rescaled dimensionless temperature, and $c_{n}$ $=n$ the Matsubara modes in the bosonic case, Eq. (4.3). The constants $v_{d}$ are defined in Eq. (3.4).

The leading order behavior of Eq. (A1) is given by the function

$$
l_{0}^{d}(\omega)=\frac{2}{d} \frac{1}{1+\omega}
$$

which follows from Eq. (A1) for $z=1, \tau=0$ and $\eta=0$. Factorizing the main building block Eq. (A2) from Eq. (A1), we notice that the remaining factor depends on both $\omega$ and the variable $z$ only through the combination

$$
\xi \equiv \frac{1-z}{1+\omega} .
$$

Therefore, it is most natural to make the variable transform Eq. (A3) by writing $l_{0}^{d}(\omega, z, \tau, \eta) \equiv l_{0}^{d}(\omega, \xi(z, \omega), \tau, \eta)$, and to rewrite the flow $(\mathrm{A} 1)$ as

$$
l_{0}^{d}(\omega, \xi, \tau, \eta)=l_{0}^{d}(\omega) B_{d}(\xi, \tau, \eta),
$$

where

$$
\begin{aligned}
B_{d}(\xi, \tau, \eta)= & \frac{d}{2} \frac{v_{d-1}}{v_{d}} \frac{\tau}{2 \pi} \sum_{n} \int_{0}^{1-c_{n}^{2} \tau^{2}} d y y^{(d / 2)-(3 / 2)} \\
& \times \frac{1-\frac{\eta}{2}\left(1-y-c_{n}^{2} \tau^{2}\right)}{1-\xi\left(y+c_{n}^{2} \tau^{2}\right)} \Theta\left(1-c_{n}^{2} \tau^{2}\right) .
\end{aligned}
$$

Below, if not stated otherwise, we adopt a simplified notation: functions are evaluated at the points $\xi=0, z=1, \tau$ $=0$ or $\eta=0$, if the corresponding arguments are not displayed. With these definitions at hand, we can face the explicit computation of Eq. (A1).

Let us compute the function $B_{d}(\xi, \tau, \eta)$ more explicitly. Since the anomalous dimension enters only linearly in Eq. (A1), it is helpful to rewrite Eq. (A5) as

$$
B_{d}(\xi, \tau, \eta)=B_{d}(\xi, \tau)-\eta \bar{B}_{d}(\xi, \tau) .
$$

For $\xi<1$, the remaining integration over the momentum variable in Eq. (A5) can be performed. This leads to

$$
\begin{aligned}
B_{d}(\xi, \tau)= & \frac{d}{d-1} \frac{v_{d-1}}{v_{d}} \frac{\tau}{2 \pi} \sum_{n} A_{n}(\xi, \tau)\left(1-c_{n}^{2} \tau^{2}\right)^{(d-3) / 2} \\
& \times \Theta\left(1-c_{n}^{2} \tau^{2}\right)_{2} F_{1}\left(1, \frac{d-1}{2} ; \frac{d+1}{2} ; \xi A_{n}(\xi, \tau)\right) .
\end{aligned}
$$

The function

$$
\begin{aligned}
{ }_{2} F_{1}(a, b ; c ; z)= & \frac{\Gamma(c)}{\Gamma(b) \Gamma(c-b)} \int_{0}^{1} d t t^{b-1}(1-t)^{c-b-1} \\
& \times(1-t z)^{-a}
\end{aligned}
$$

with ${ }_{2} F_{1}(a, b ; c ; z)={ }_{2} F_{1}(b, a ; c ; z)$ denotes the hypergeometric function [41]. We also introduced the thermal amplitude function

$$
A_{n}(\xi, \tau)=\frac{1-c_{n}^{2} \tau^{2}}{1-\xi c_{n}^{2} \tau^{2}} .
$$

The index $n$ corresponds to the Matsubara mode. The factors $A_{n}$ only appear in combination with the factor $\Theta\left(1-c_{n}^{2} \tau^{2}\right)$. At the limits,

$$
\begin{aligned}
A_{n}(\xi, 0) & =A_{0}(\xi, \tau)=1 \\
A_{n}\left(\xi, c_{n}^{-1}\right) & =0 .
\end{aligned}
$$

In the same way, we find from Eq. (A5) for the term $\sim \eta$ in Eq. (A4) the explicit expression

$$
\begin{aligned}
\bar{B}_{d}(\xi, \tau)= & \frac{1}{2} \frac{d}{d-1} \frac{v_{d-1}}{v_{d}} \frac{\tau}{2 \pi} \sum_{n} A_{n}(\xi, \tau) \\
& \times\left(1-c_{n}^{2} \tau^{2}\right)^{(d-1) / 2} \Theta\left(1-c_{n}^{2} \tau^{2}\right) \\
& \times\left[{ }_{2} F_{1}\left(1, \frac{d-1}{2} ; \frac{d+1}{2} ; \xi A_{n}(\xi, \tau)\right)\right. \\
& \left.-\frac{d-1}{d+1}{ }_{2} F_{1}\left(1, \frac{d+1}{2} ; \frac{d+3}{2} ; \xi A_{n}(\xi, \tau)\right)\right] .
\end{aligned}
$$

Combining Eq. (A7) with Eq. (A12) gives Eq. (A6) and hence Eq. (A4) explicitly. Equation (A4) is the most general expression for the factorization at second order in the derivative expansion and at finite temperature.

The temperature dependence of the function $B_{d}(\xi, \tau, \eta)$ describes dimensional reduction. In particular, it obeys the limits

$$
\begin{aligned}
& B_{d}(\xi, \tau=0, \eta) \equiv B_{d}(\xi, \eta) \\
& B_{d}(\xi, \tau \geqslant 1, \eta)=\frac{v_{d-1}}{v_{d}} \frac{d}{d-1} \frac{\tau}{2 \pi} B_{d-1}(\xi, \eta) .
\end{aligned}
$$

The low- and high-temperature limits (A13) and (A14) are discussed in the following Appendixes. 
Let us consider the case where $\xi=0$. It corresponds either to the case of a field-independent wave function renormalization $z \equiv 1$, and/or to the decoupling regime $\omega \gg 1$. A few properties of Eq. (A4) have been discussed in the main text. For $\xi=0$ the factor (A6) reduces to

$$
\begin{aligned}
B_{d}(\tau, \eta)= & \frac{d}{d-1} \frac{v_{d-1}}{v_{d}} \frac{\tau}{2 \pi} \sum_{n}\left(1-c_{n}^{2} \tau^{2}\right)^{(d-1) / 2} \\
& \times\left(1-\eta \frac{1-c_{n}^{2} \tau^{2}}{d+1}\right) \Theta\left(1-c_{n}^{2} \tau^{2}\right)
\end{aligned}
$$

This corresponds to Eq. (4.13) discussed in Sec. IV. In addition, we notice that

$$
\begin{aligned}
& B_{d}(\tau, \eta \rightarrow 0)=B_{d}(\tau) \\
& B_{d}(\tau \rightarrow 0, \eta)=1-\frac{\eta}{d+2} \\
& B_{d}(\tau \geqslant 1, \eta)=\frac{d}{d-1} \frac{v_{d-1}}{v_{d}} \frac{\tau}{2 \pi}\left(1-\frac{\eta}{d+1}\right) .
\end{aligned}
$$

Equation (A16) corresponds to Eq. (3.9), Eq. (A17) to the low-temperature limit (5.15), and Eq. (A18) to the hightemperature limit (4.15).

Similar results are found for the fermionic case, though not discussed explicitly.

\section{APPENDIX B: LOW TEMPERATURE LIMIT}

In the low temperature limit $\tau \rightarrow 0$, the flow (A1) simplifies to

$$
l_{0}^{d}(\omega, z, \eta)=\int_{0}^{1} d y y^{(d-2) / 2} \frac{1-\frac{1}{2} \eta(1-y)}{(z-1) y+1+\omega} .
$$

The remaining integration in Eq. (B1) is solved to give

$$
l_{0}^{d}(\omega, \xi, \eta)=l_{0}^{d}(\omega) B_{d}(\xi, \eta)
$$

with

$$
B_{d}(\xi, \eta)=\frac{d}{2} \int_{0}^{1} d y y^{(d / 2)-1}\left(\frac{1}{1-\xi y}-\frac{\eta}{2} \frac{1-y}{1-\xi y}\right)
$$

in arbitrary dimensions. For $\xi \equiv(1-z) /(1+\omega)<1$, and hence $z>-\omega$, the integration can be peformed analytically. We find

$$
B_{d}(\xi, \eta)=B_{d}(\xi)-\eta \bar{B}_{d}(\xi)
$$

where

$$
B_{d}(\xi)={ }_{2} F_{1}\left(1, \frac{d}{2} ; 1+\frac{d}{2} ; \xi\right)
$$

and

$$
\begin{aligned}
\bar{B}_{d}(\xi)= & \frac{1}{2}\left[{ }_{2} F_{1}\left(1, \frac{d}{2} ; 1+\frac{d}{2} ; \xi\right)\right. \\
& \left.-\frac{d}{d+2}{ }_{2} F_{1}\left(1,1+\frac{d}{2} ; 2+\frac{d}{2} ; \xi\right)\right] .
\end{aligned}
$$

For $|\xi|<1$, Eq. (B4) can be Taylor-expanded in $\xi$, leading to the first equation given in Eq. (5.14).

For applications, it will be useful to obtain explicit analytical expressions for the functions (B1) for fixed dimensions. For $d=4$, we find

$$
\begin{aligned}
l_{0}^{4}(\omega, z, \eta)= & \frac{1}{z-1}-\frac{1+\omega}{(z-1)^{2}} \ln \frac{z+\omega}{1+\omega} \\
& -\eta\left[\frac{1+2 \omega+z}{4(z-1)^{2}}-\frac{1}{2} \frac{(1+\omega)(\omega+z)}{(z-1)^{3}} \ln \frac{z+\omega}{1+\omega}\right] .
\end{aligned}
$$

In $d=3$ dimensions, we find

$$
\begin{aligned}
l_{0}^{3}(\omega, z, \eta)= & \frac{2}{(z-1)}-\frac{2 \sqrt{1+\omega}}{(z-1)^{3 / 2}} \arctan \sqrt{\frac{z-1}{1+\omega}} \\
& -\eta\left[\frac{1+3 \omega+2 z}{3(z-1)^{2}}\right. \\
& \left.-\sqrt{\frac{1+\omega}{z-1}} \frac{z+\omega}{(z-1)^{2}} \arctan \sqrt{\frac{z-1}{1+\omega}}\right]
\end{aligned}
$$

and the region for $z<1$ is obtained through analytical continuation. Finally, for $d=2$ we find

$$
\begin{aligned}
l_{0}^{2}(\omega, z, \eta)= & \frac{1}{z-1} \ln \frac{z+\omega}{1+\omega}-\eta\left[-\frac{1}{2} \frac{1}{z-1}\right. \\
& \left.+\frac{z+\omega}{2(z-1)^{2}} \ln \frac{z+\omega}{1+\omega}\right] .
\end{aligned}
$$

Notice that the functions (B7), (B8) and (B9) behave smoothly for $z \approx 1$, which follows either from Eq. (5.14) or by an explicit check.

For $z=1$ these expressions reduce to the result Eq. (5.15).

\section{APPENDIX C: HIGH TEMPERATURE LIMIT}

The high-temperature limit is reached for $\tau \geqslant 1$, or $T$ $\geqslant 2 \pi k$. Then, only the $n=0$ Matsubara mode contributes to the flow. Using Eqs. (A7), (A10) and (B5), we find:

$$
B_{d}(\xi, \tau \geqslant 1, \eta)=\frac{d}{d-1} \frac{v_{d-1}}{v_{d}} \frac{\tau}{2 \pi} B_{d-1}(\xi, \tau=0, \eta) .
$$

For $|\xi|<1$, Eq. (C1) can be Taylor-expanded as 


$$
\begin{aligned}
B_{d}(\xi, \tau \geqslant 1, \eta)= & \frac{d}{d-1} \frac{v_{d-1}}{v_{d}} \frac{\tau}{2 \pi} \sum_{n=0}^{\infty} \frac{(d-1) \xi^{n}}{2 n+d-1} \\
& \times\left(1-\frac{\eta}{d+1+2 n}\right) .
\end{aligned}
$$

Splitting $B_{d}(\xi, \tau \geqslant 1, \eta)$ as in Eq. (A6), we have

$$
B_{d}(\xi, \tau \geqslant 1)=\frac{d}{d-1} \frac{v_{d-1}}{v_{d}} \frac{\tau}{2 \pi}{ }_{2} F_{1}\left(1, \frac{d-1}{2} ; \frac{d+1}{2} ; \xi\right) .
$$

In turn, the flow proportional to the anomalous dimension reduces to

$$
\begin{aligned}
\bar{B}_{d}(\xi, \tau \geqslant 1)= & \frac{1}{2} \frac{d}{d-1} \frac{v_{d-1}}{v_{d}} \frac{\tau}{2 \pi} \times \\
& \times\left[{ }_{2} F_{1}\left(1, \frac{d-1}{2}, \frac{d+1}{2}, \xi\right)\right. \\
& \left.-\frac{d-1}{d+1}{ }_{2} F_{1}\left(1, \frac{d+1}{2}, \frac{d+3}{2}, \xi\right)\right] .
\end{aligned}
$$

[1] K.G. Wilson and I.G. Kogut, Phys. Rep. 12, 75 (1974).

[2] F.J. Wegner and A. Houghton, Phys. Rev. A 8, 401 (1973).

[3] J. Polchinski, Nucl. Phys. B231, 269 (1984).

[4] C. Wetterich, Phys. Lett. B 301, 90 (1993).

[5] U. Ellwanger, Z. Phys. C 62, 503 (1994).

[6] T.R. Morris, Int. J. Mod. Phys. A 9, 2411 (1994).

[7] C. Bagnuls and C. Bervillier, Phys. Rep. 348, 91 (2001).

[8] J. Berges, N. Tetradis, and C. Wetterich, hep-ph/0005122.

[9] R.D. Ball, P.E. Haagensen, J.I. Latorre, and E. Moreno, Phys. Lett. B 347, 80 (1995).

[10] D.F. Litim, Phys. Lett. B 393, 103 (1997).

[11] K. Aoki, K. Morikawa, W. Souma, J. Sumi, and H. Terao, Prog. Theor. Phys. 99, 451 (1998).

[12] S. Liao, J. Polonyi, and M. Strickland, Nucl. Phys. B567, 493 (2000).

[13] F. Freire and D.F. Litim, Phys. Rev. D 64, 045014 (2001).

[14] J. Sumi, W. Souma, K. Aoki, H. Terao, and K. Morikawa, hep-th/0002231.

[15] D.F. Litim, Phys. Lett. B 486, 92 (2000).

[16] J.I. Latorre and T.R. Morris, J. High Energy Phys. 11, 004 (2000).

[17] P.M. Stevenson, Phys. Rev. D 23, 2916 (1981).

[18] D.F. Litim, Int. J. Mod. Phys. A 16, 2081 (2001).

[19] T. Appelquist and J. Carazzone, Phys. Rev. D 11, 2856 (1975).

[20] S. Liao, Phys. Rev. D 53, 2020 (1996).

[21] D.F. Litim and J.M. Pawlowski, Phys. Lett. B 516, 197 (2001).

[22] J. Alexandre, V. Branchina, and J. Polonyi, Phys. Lett. B 445, 351 (1999).
[23] S. Bornholdt and C. Wetterich, Z. Phys. C 58, 585 (1993).

[24] D.U. Jungnickel and C. Wetterich, Phys. Rev. D 53, 5142 (1996).

[25] A. Hasenfratz and P. Hasenfratz, Nucl. Phys. B270, 687 (1986).

[26] N. Tetradis and D.F. Litim, Nucl. Phys. B464, 492 (1996).

[27] J. Comellas and A. Travesset, Nucl. Phys. B498, 539 (1997).

[28] T.R. Morris, Phys. Lett. B 329, 241 (1994).

[29] G.R. Golner, Phys. Rev. B 33, 7863 (1986).

[30] C. Wetterich, Z. Phys. C 57, 451 (1993).

[31] N. Tetradis and C. Wetterich, Nucl. Phys. B383, 197 (1992).

[32] N. Tetradis and C. Wetterich, Nucl. Phys. B422, 541 (1994).

[33] D.F. Litim and N. Tetradis, hep-th/9501042.

[34] B. Bergerhoff, F. Freire, D.F. Litim, S. Lola, and C. Wetterich, Phys. Rev. B 53, 5734 (1996); B. Bergerhoff, D.F. Litim, S. Lola, and C. Wetterich, Int. J. Mod. Phys. A 11, 4273 (1996).

[35] N. Tetradis and C. Wetterich, Nucl. Phys. B398, 659 (1993).

[36] D.F. Litim, "Wilsonian flow equation and thermal field theory," hep-ph/9811272.

[37] J. Schwinger, Phys. Rev. 82, 664 (1951).

[38] M. Oleszczuk, Z. Phys. C 64, 533 (1994).

[39] For a recent review, see D.F. Litim and J.M. Pawlowski, "On gauge invariant Wilsonian flows," hep-th/9901063, and references therein.

[40] F.J. Wegner, Ann. Phys. (Leipzig) 3, 77 (1994).

[41] Higher Transcendental Functions, edited by A. Erdélyi (McGraw-Hill, New York, 1953), Vol. 1. 